\title{
Nonlinear radiative cooling of relativistic particles under equipartition conditions
}

\section{Instantaneous monoenergetic injection}

\author{
R. Schlickeiser and I. Lerche \\ Institut für Theoretische Physik, Lehrstuhl IV: Weltraum- und Astrophysik, Ruhr-Universität Bochum, 44780 Bochum, Germany \\ e-mail: rsch@tp4.ruhr-uni-bochum.de
}

Received 14 June 2007 / Accepted 4 September 2007

\begin{abstract}
Context. In powerful cosmic nonthermal radiation sources with dominant magnetic-field self generation, the generation of magnetic fields at almost equipartition strength by relativistic plasma instabilities operates as fast as the acceleration or injection of ultra-high energy radiating electrons and hadrons in these sources. Consequently, the magnetic field strength becomes time-dependent and adjusts itself to the actual kinetic energy density of the radiating electrons in these sources. This coupling of the magnetic field and the magnetic field energy density to the kinetic energy of the radiating particles changes both the synchrotron emissivity and the intrinsic temporal evolution of the relativistic particle energy spectrum after injection.

Aims. The nonlinear kinetic equation for the intrinsic temperoral evolution of relativistic electrons is solved for the case of instantaneous injection of monoenergetic particles.

Methods. Analytical derivations and graphical illustrations.

Results. In blazar and gamma-ray burst sources, the nonlinear synchrotron cooling of each particle under equipartition conditions is then orders of magnitude quicker than the linear cooling behaviour in constant magnetic-field strength sources. This dramatic reduction for the intrinsic radiation loss time may be essential for understanding the observed rapid time variation, of the order of days in the case of the non-blazar radio galaxy M 87 and minutes in flaring blazar jets such as PKS 2155-304. Significant differences in the optically-thin, synchrotron spectral distributions at different times and in the synchrotron light curves at different frequencies are predicted.
\end{abstract}

Key words. radiation mechanisms: non-thermal - galaxies: active - cosmic rays - magnetic fields

\section{Introduction}

The broadband photon spectra of powerful nonthermal sources of radiation, such as blazars, exhihit two broad spectral components. As the extreme class of relativistic jets from active galactic nuclei, blazars are characterised by rapid variability at all wavelengths and a high degree of linear polarization in the optical. While there is considerable debate about the nature of the high-energy spectral component dependent on an hadronic or leptonic particle acceleration model in these sources, it is agreed that the low-energy component is nonthermal radiation from high-energy relativistic electrons (for a recent review see Böttcher 2007). Most researchers favour synchrotron radiation although alternatives have been proposed, such as electrostatic bremsstrahlung (Schlickeiser 2003) in the case of blazars and Jitter radiation in the case of GRB afterglows (Medvedev et al. 2007; Workman et al. 2007). Synchrotron or Jitter radiation is also regarded as a prime mechanism generating the nonthermal radiation from the afterglows of gamma-ray burst (GRB) sources (Meszaros \& Rees 1997; Piran 1999; Waxman 2006).

In all three radiation processes, the electron radiative loss time is so short that considerable spectral evolution of the energy spectrum of the radiating electrons occurs, controlled by the energy density in the magnetic fields (for synchrotron and Jitter radiation) or the electrostatic plasma turbulence (for electrostatic bremsstrahlung). Electron spectral evolution calculations mostly follow the pioneering work of Kardashev (1962) addressing the temporal evolution of the volume-averaged differential density $N(p, t)$ of relativistic electrons for different assumed injection scenarios.

Numerical modelling of the observed blazar spectral energy distributions (Dermer \& Schlickeiser 2002; Böttcher \& Chiang 2002) provides the best agreement if equipartition conditions are taken between the energy densities of magnetic fields $\left(U_{B}=B^{2} / 8 \pi\right)$ and relativistic electrons,

$U_{\mathrm{e}}(t)=\int_{0}^{\infty} \mathrm{d} p \gamma m_{\mathrm{e}} c^{2} N(p, t)$,

i.e. constant values of the equpartition parameter $e_{B}=$ $U_{B}(t) / U_{\mathrm{e}}(t)$. For powerful flat-spectrum radio quasars, equipartition values of order unity are inferred, while in TeV BL-Lacs sub-equipartition fields $0.01<e_{B}<0.1$ have been derived (Kino et al. 2002). Consequently, the magnetic field strength becomes time-dependent,

$B(t)=\sqrt{8 \pi e_{B} U_{\mathrm{e}}(t)}$

adjusting itself to the actual kinetic energy density of the radiating particles. 
A similar equipartition assumption between the magnetic and particle energy densities is also made in the nonthermal radiation models for gamma-ray burst afterglows (Meszaros \& Rees 1993, 1997; Paczynski \& Rhoads 1993; Frail et al. 2000). Here the cosmological fireball model is a spherical blast wave expanding adiabatically into a homogeneous medium. A fixed fraction $\epsilon_{\mathrm{e}}$ of the blast-wave energy $E_{0}$, dominated by hadrons, goes into accelerating a power-law distribution of electrons above a lower ultrarelativistic cut-off $\gamma_{m}$. In the presence of a magnetic field, which itself is a fixed fraction $\epsilon_{B}$ of the energy density of the blast wave, the electrons emit synchrotron radiation. The ratio $\epsilon_{B} / \epsilon_{\mathrm{e}}=e_{B}$ of the fixed fractions thus reflects the equipartition condition in the emission region (Sari et al. 1998; Frail et al. 2000; Van der Horst et al. 2007). In the case of gamma-ray bursts, the data fits to observed spectra generally lead to different values of the equipartition parameters of the electrons $\left(\epsilon_{\mathrm{e}}\right)$ and of the magnetic fields $\left(\epsilon_{B}\right)$ compared to the blast-wave energy hence to a different energy in electrons and magnetic fields i.e. $e_{B} \neq 1$ (van Paradijs et al. 2000; Panaitescu \& Kumar 2000; Piran 2005).

Equipartition conditions are often invoked in astrophysical sources for convenience, as discussed e.g. in the review by Beck \& Krause (2005). Observationally, for a variety of nonthermal sources, the equipartition concept is supported by magnetic field estimates as e.g. the Coma cluster of galaxies (Schlickeiser et al. 1988). From a theoretical point of view, the coupling of the processes of particle acceleration and magnetic field generation in collision-poor astrophysical plasmas should lead to some kind of near equipartition. For example, equipartition conditions are expected to hold if the magnetic field in the radiation sources is produced by the interaction of the relativistic jet (in the case of blazars) or the external shock (in the case of GRBs) with the surrounding ambient interstellar or intergalactic medium (Stockem et al. 2007). Both interactions are prominent examples of the relativistic collision of plasma shells with different properties (temperature, density, composition, etc.). Experimentally (Kapetanakos 1974; Tatarakis et al. 2003) and from numerous particle-in-cell (PIC) simulations (e.g. Lee \& Lampe 1973; Nishikawa et al. 2003; Silva et al. 2003; Frederiksen et al. 2004; Sakai et al. 2004; Jaroschek et al. 2005), such collisions of plasma shells lead to the onset of linear Weibel-type plasma instabilities perpendicular to the flow directions in both unmagnetised and slightly magnetised plasmas, and subsequently to the development of nonlinear structures such as solitons, shocks, and double layers (Moiseev \& Sagdeev 1963; Montgomery \& Joyce 1969; Schamel 1972; Medvedev \& Loeb 1999). The PIC simulations of electron-proton and electronpositron plasmas demonstrate that these instabilities generate magnetic fields in the form of aperiodic fluctuations at almost equipartition ( $e_{B} \simeq 0.1$ typically for electron-proton plasmas) strength on the shortest plasma time scale. The aperiodic magnetic fluctuations will isotropise the initially anisotropic particle distributions in the rest frame of the jet leading to an efficient pick-up of nearly monoenergetic relativistic electrons and protons (Schlickeiser et al. 2002). On similar time scales, ultrarelativistic particles are energised in these structures. Moreover, on comparitively longer time scales, energetic electrons are accelerated in the shock structures to ultra-high energies. Therefore nonlinear cooling of the relativistic electrons is particularly relevant for astrophysical sources with dominant magnetic-field self generation.

However, the PIC simulations usually show a tendency towards equipartition between a quasi-thermal distribution of electrons and the fields. Many simulations do not run long enough, or over enough plasma lengths to show a clear non-thermal electron distribution. However, the recent PIC simulation by $\mathrm{Ng}$ $\&$ Noble (2006) indicates that, when the Weibel instability occurs, an inductive Faraday acceleration mechanism can effectively power nonthermal energetic particles due to electrostatic plasma wakefields. These electrostatic wakefields are excited by an initially charge-neutral jet when oppositely charged current filaments separate.

In the following we concentrate on the synchrotron radiation of relativistic electrons, although our considerations also apply to the synchrotron radiation of heavier, charged particles such as hadrons (Reimer et al. 2004) and muons (Böttcher \& Reimer 2004). Because the Thomson cross section of hadrons of charge $Z e$ and mass $A m_{\mathrm{p}}$,

$\sigma_{\mathrm{H}}=\frac{Z^{4}}{A^{2}}\left(\frac{m_{\mathrm{e}}}{m_{\mathrm{p}}}\right)^{2} \sigma_{\mathrm{T}}=2.96 \times 10^{-7} \sigma_{\mathrm{T}}$,

is more than six orders of magnitude smaller than the electron Thomson cross section $\sigma_{\mathrm{T}}=6.65 \times 10^{-25} \mathrm{~cm}^{2}$, hadron or muon synchrotron radiation in general - besides large particle Lorentz factors - requires particularly strong magnetic field values, exactly the conditions provided if equipartition conditions with the radiating particles' energy density hold.

Here we study the consequences of the time-dependences of $U_{B}(t)$ and $B(t)$ for non-steady sources because:

1. Under equipartition (or better partition) conditions, the synchrotron cooling of relativistic electrons depends on the kinetic energy density of the radiating particles, which, according to Eq. (1), is an integral over the electron differential spectral density. As a consequence, the evolution of relativistic electron energy spectra exhibits nonlinear behaviour because the cooling of an individual electron is stronger with a higher kinetic energy density of the whole electron population.

2. Under equipartition conditions, the magnetic field strength (2) is time-dependent, changing not only the temporal behaviour of the relativistic electron energy spectrum but, additionally, modifying the synchrotron photon emissivity.

For simplicity, we illustrate the results for the case of an instantaneous injection of monoenergetic electrons. More complex injection scenarios (e.g. instantaneous or extended injection of power laws) complicate the mathematical solution of the nonlinear kinetic equation and will be considered elsewhere. We also perform the calculations explicitly for the case of synchrotron cooling and radiation. However, (i) as Jitter radiation losses and electrostatic bremsstrahlung losses also exhibit a quadratic dependence on the electron Lorentz factor, and (ii) because the energy densities of the mean magnetic field (in the case of Jitter radiation) and of the electrostatic plasma waves (in the case of electrostatic bremsstrahlung) also are subject to equipartition with the relativistic electrons, a similar nonlinear cooling behaviour is expected for these alternative radiation processes.

After identifying the basic equations, we highlight the differences in the electron energy spectra between the two cooling regimes and calculate the differences in the produced synchrotron radiation spectra. All mathematical details can be found in the appendices.

\section{Linear and nonlinear electron cooling}

All physical quantities are calculated in a coordinate system comoving with the radiation source. The energy loss rate of 
relativistic electrons due to synchrotron radiation in a large-scale random magnetic field is

$|\dot{\gamma}|=D_{0} \gamma^{2}$

with the rate

$D_{0}=\frac{4}{3} \frac{c \sigma_{\mathrm{T}}}{m_{\mathrm{e}} c^{2}} U_{B}=2.66 \times 10^{-14}\left[\frac{U_{B}}{m_{\mathrm{e}} c^{2}}\right] \mathrm{s}^{-1}$

where $\gamma$ is the electron Lorentz factor, $c$ denotes the speed of light, and $\sigma_{\mathrm{T}}=6.65 \times 10^{-25} \mathrm{~cm}^{2}$ is the Thomson cross section. The competition between the instantaneous injection of ultrarelativistic electrons $\left(\gamma_{0} \gg 1\right)$, at the rate $Q(\gamma, t)=q_{0} \delta\left(\gamma-\gamma_{0}\right) \delta(t)$ at time $t=0$, and the electron synchrotron energy losses implies for a time-dependent evolution of the volume-averaged relativistic electron population inside the radiating source (Kardashev 1962) given through

$\frac{\partial n(\gamma, t)}{\partial t}-\frac{\partial}{\partial \gamma}[|\dot{\gamma}| n(\gamma, t)]=q_{0} \delta\left(\gamma-\gamma_{0}\right) \delta(t)$

where $n(\gamma, t)$ is the volume-averaged differential number density. Throughout we consider ultrarelativistic electrons $(\gamma \gg 1)$ so that the relation $p \simeq m_{\mathrm{e}} c \gamma$ is appropriate, implying the relation $N(p, t)=n(\gamma, t) / m_{\mathrm{e}} c$ between the respective differential electron densities. The kinetic energy density in relativistic electrons then is $U_{\mathrm{e}}(t)=m_{\mathrm{e}} c^{2} \int_{0}^{\infty} \mathrm{d} \gamma \gamma n(\gamma, t)$. Using this ultrarelativistic approximation is also mathematically very convenient, as all $\gamma$-integrals are taken from zero to infinity.

In the case of linear cooling with constant energy density $U_{B}$, the rate (5) is constant, and the solution of the kinetic Eq. (6) is (for details see Appendix A)

$n_{L}\left(\gamma, \gamma_{0}\right)=q_{0} H\left[\gamma_{0}-\gamma\right] \delta\left(\gamma-\gamma_{L}(t)\right)$

where $H[x]$ denotes Heaviside's step function and

$\gamma_{L}(t)=\frac{\gamma_{0}}{1+D_{0} \gamma_{0} t}$.

An electron starting with Lorentz factor $\gamma_{0}$ has cooled to the Lorentz factor $\gamma_{L}$ at later times. The half-life time, $t_{1 / 2}^{L}$, where the Lorentz factor has cooled to half its initial value $\gamma_{L}\left(t_{1 / 2}^{L}\right)=\gamma_{0} / 2$,

$t_{1 / 2}^{L}=\frac{1}{D_{0} \gamma_{0}}=\frac{3.76 \times 10^{13}}{\gamma_{0}}\left[\frac{4.54 \mathrm{mG}}{B}\right]^{2} \mathrm{~s}$,

depends inversely on the initial Lorentz factor and the magnetic field strength. In particular, $t_{1 / 2}^{L}$ does not depend on the strength $q_{0}$ of the injection rate.

Under equipartition conditions, the magnetic-field energy density $U_{B}(t)=e_{B} U_{\mathrm{e}}(t)=e_{B} m_{\mathrm{e}} c^{2} \int_{0}^{\infty} \mathrm{d} \gamma \gamma n(\gamma, t)$ depends on an energy integral of the actual electron spectrum, so that the loss rate (4) is

$|\dot{\gamma}|=A_{0} \gamma^{2} \int_{0}^{\infty} \mathrm{d} \gamma \gamma n(\gamma, t)$

with the abbreviation $A_{0}=\frac{4}{3} c \sigma_{\mathrm{T}} e_{B} \mathrm{~cm}^{3} \mathrm{~s}^{-1}$. In this case the solution of the nonlinear kinetic equation is (Appendix B)

$n_{N L}\left(\gamma, \gamma_{0}, t\right)=q_{0} H\left[\gamma_{0}-\gamma\right] \delta\left(\gamma-\gamma_{N L}(t)\right)$

where

$\gamma_{N L}(t)=\frac{\gamma_{0}}{\sqrt{1+2 A_{0} q_{0} t \gamma_{0}^{2}}}$.
Equation (12) with $e_{B}=e_{B}(0)=U_{B}(0) / U_{\mathrm{e}}(0)$ implies the modified synchrotron energy loss rate in equipartition conditions

$$
\begin{aligned}
\left|\gamma_{\dot{N} L}\right| & =A_{0} q_{0} \gamma_{N L}^{3}=\frac{4}{3} c \sigma_{\mathrm{T}} e_{B} q_{0} \gamma_{N L}^{3} \\
& =D_{0} m_{\mathrm{e}} c^{2} \frac{U_{B}(0)}{U_{\mathrm{e}}(0)} \gamma_{N L}^{3},
\end{aligned}
$$

which is now proportional to $\gamma_{N L}^{3}$.

Here, an electron starting with Lorentz factor $\gamma_{0}$ cools to the Lorentz factor $\gamma_{N L}$ at later times, but $\gamma_{N L}$ differs substantially from the linear Lorentz factor (8). Now the half-life time, $t_{1 / 2}^{N L}$, is

$$
\begin{aligned}
t_{1 / 2}^{N L} & =\frac{3}{2 A_{0} q_{0} \gamma_{0}^{2}}=\frac{9}{8 c \sigma_{\mathrm{T}} e_{B} q_{0} \gamma_{0}^{2}} \\
& =5.64 \times 10^{13} e_{B}^{-1}\left[q_{0} / \mathrm{cm}^{-3}\right]^{-1} \gamma_{0}^{-2} \mathrm{~s},
\end{aligned}
$$

which depends on the initial kinetic energy of injected electrons (proportional to $q_{0} \gamma_{0}$ ), differently than in the linear case on the initial electron Lorentz factor (inversely quadratic instead of inversely linear) and also inversely on the value of the injection rate $q_{0}$. The more electrons are injected, the quicker each electron cools under equipartition requirements. Such a collective behaviour is new and completely different from the linear case.

The ratio of nonlinear (14) to linear (9) half-life time is much smaller than unity provided $q_{0} \gamma_{0}>\frac{3}{2 e_{B}}\left[\frac{B}{4.54 \mathrm{mG}}\right]^{2}$, which is easily fulfilled in GRB sources and blazars. Modelling of blazar flaring (e.g. Dermer \& Schlickeiser 1992, 2002) indicates that about $q_{0} \simeq 10^{5}$ electrons per $\mathrm{cm}^{-3}$ with Lorentz factors $\gamma_{0} \simeq 10^{7}$ are injected in sources with several Gauss $(B \simeq 10 \mathrm{G})$ magnetic fields, much below the initial equipartition field strength. The nonlinear half-life is then more than four orders of magnitude shorter than the standard linear half-life.

This dramatic reduction of the intrinsic radiation loss times due to equipartition conditions meets the drastic requirements on the required time dilatation from Doppler boosting to explain the observed short time variability from flaring blazar jets, close to minutes as in the flare of PKS 2155-304 on 2006 July 28 (Aharonian et al. 2007) and GRBs (of order seconds). It also offers an explanation of the observed rapid time variation (of days) in the case of the non-blazar radio galaxy M 87 (Aharonian et al. 2006). These observations predominantly constrain the size $R$ of the emitting source by $t_{\mathrm{var}} \simeq R / c \delta$, where $\delta$ is the Doppler factor, However, the intrinsic cooling time of the radiating electrons then also has to be smaller than $R / c$; otherwise, the combination of the short light travel time with the larger electron cooling time would lead to a variability time scale that is larger than observed. For radiating electrons, the observed cooling time scales of hundreds of seconds can be easily achieved even with conditions close to that associated to the standard parameters derived for these sources. If the high-energy gamma radiation stems from hadron synchrotron radiation, one would, however, need the dramatic reduction of the intrinsic radiation loss times due to equipartition conditions.

In the specific case of the flare of PKS 2155-304 on 2006 July 28, the quasi-simultaneous spectral energy distribution provided by the Swift data reported in Foschini et al. (2007) suggests that the main gamma-ray emission channel seems to be the inverse Compton process (probably synchrotron self-Compton emission), whereas synchrotron radiation provides the photons in the optical, UV, and X-ray bands. Our results on the nonlinear cooling of the electrons also apply here. The synchroton-self Compton process is modified because the synchrotron photon target gas evolves nonlinearly with time. 
Therefore the SSC energy loss rate of a single electron is different than in the linear cooling case. In the case of external Compton scattering, intrinsic synchrotron losses dominate external Compton cooling due to the different Doppler boosting factors (Dermer \& Schlickeiser 2002). The external Compton scattering process would be a suitable candidate for the additional gamma-ray emission process that, according to Foschini et al. (2007), is necessary to account for the difference in the observed minute variability in the $\mathrm{TeV}$ band as compared to the typically observed hour variability in the X-ray band (Zhang et al. 2002).

It is easy to show that, with assumed equipartition conditions $U_{B}(0)=q_{0} \gamma_{0} m_{\mathrm{e}} c^{2} e_{B}$, the linear (9) half-life time is comparable to the nonlinear (14) half-life time, $t_{1 / 2}^{L}=(2 / 3) t_{1 / 2}^{N L}$.

\section{Intrinsic optically thin synchrotron radiation}

The pitch-angle averaged synchrotron power of a single electron is (Crusius \& Schlickeiser 1986)

$P(v, \gamma)=P_{0} \frac{v}{\gamma^{2}} C S\left[\frac{2 v}{3 v_{0} \gamma^{2}}\right]$

with $P_{0}=8.763 \times 10^{-29} \mathrm{erg} \mathrm{s}^{-1} \mathrm{~cm}^{-3}$ and the electron gyrofrequency $v_{0}=e B /\left(2 \pi m_{\mathrm{e}} c\right)$. The function $C S(x)$ is approximated well by

$C S(x) \simeq \frac{x^{-2 / 3}}{0.869+x^{1 / 3} \mathrm{e}^{x}}$.

\subsection{Optically thin synchrotron intensities}

In a homogenous source of diameter $L$, the optically thin synchotron intensity from relativistic electrons with the volumeaveraged differential density $n(\gamma, t)$ then is

$I(v, t)=\frac{L}{4 \pi} \int_{0}^{\infty} \mathrm{d} \gamma n(\gamma, t) P(v, \gamma)$.

Introducing the normalized time coordinates $x_{L}=t / t_{1 / 2}^{L}$ and $x_{N L}=3 t / t_{1 / 2}^{N L}$ with the respective half-lifes, the normalized frequencies

$f_{L} \equiv \frac{v}{v_{L}(t=0)}=\frac{v}{\frac{3}{2} v_{0} \gamma_{0}^{2}}$

$f_{N L} \equiv \frac{v}{v_{N L}(t=0)}=\frac{v}{\frac{3}{2} c \sqrt{2 e_{B} q_{0} r_{0} / \pi} \gamma_{0}^{5 / 2}}$,

and the combined variables

$s_{L} \equiv f_{L}\left(1+x_{L}\right)^{2}, \quad s_{N L}=f_{N L}\left(1+x_{N L}\right)^{5 / 4}$.

As shown in Appendix C, the optically thin synchrotron intensities can then be written in the compact forms

$I_{L}\left(f_{L}, x_{L}\right)=H_{0} s_{L} C S\left[s_{L}\right] \simeq \frac{H_{0}}{0.869 s_{L}^{-1 / 3}+\mathrm{e}^{s_{L}}}$

and

$I_{N L}\left(f_{N L}, x_{N L}\right)=\frac{J_{0}}{\left(1+x_{N L}\right)^{1 / 4}} s_{N L} C S\left[s_{N L}\right]$

$\simeq \frac{J_{0}}{\left(1+x_{N L}\right)^{1 / 4}\left[0.869 s_{N L}^{-1 / 3}+\mathrm{e}^{s_{N L}}\right]}$

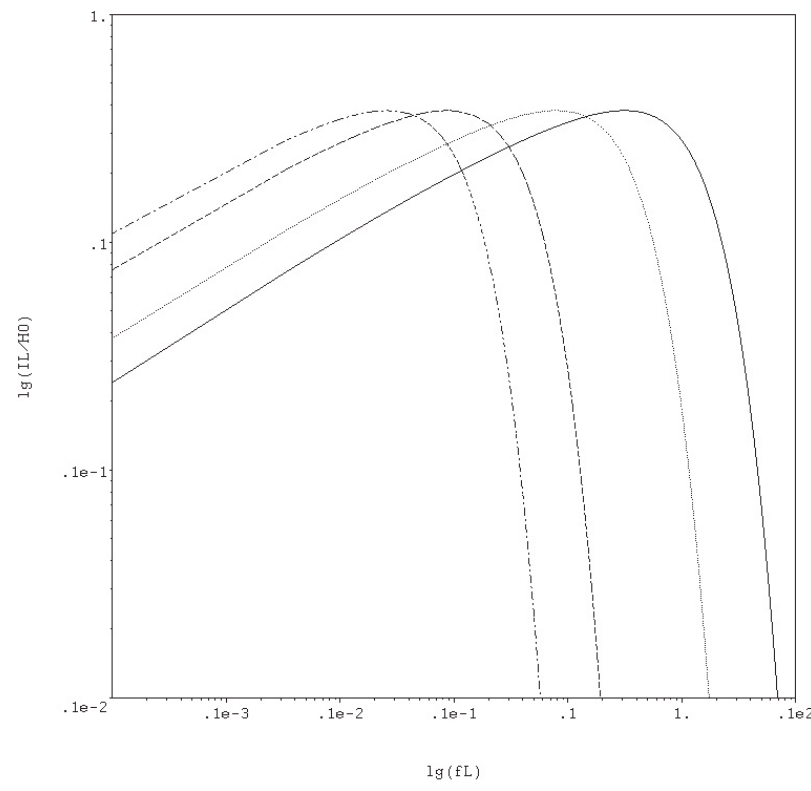

Fig. 1. Synchrotron intensity $I_{L} / H_{0}$ as a function of the normalised frequency $f_{L}$ in the linear cooling case at four different times $x_{L}=0$ (full curve, 1 st curve from right), $x_{L}=1$ (dotted curve, 2 nd curve from right), $x_{L}=5$ (dashed curve, 3 rd curve from right), and $x_{L}=10$ (dotted-dashed curve, 4 th curve from right). The maximum value of the intensity occurs at the frequency $f_{L, \max }=0.282 /\left(1+x_{L}\right)^{2}$, which decreases $\propto 0.282 x_{L}^{-2}$ for large times $x_{L} \gg 1$. The maximum intensity is independent of time due to the constant synchrotron loss rate.

with the constants

$H_{0}=\frac{3 L q_{0} P_{0} v_{0}}{8 \pi}, \quad J_{0}=\frac{3 L P_{0} q_{0} c}{8 \pi} \sqrt{2 e_{B} q_{0} r_{0} \gamma_{0} / \pi}$.

Both intensities have the same formal dependence on the variable $s$, but this variable is very different for the linear and nonlinear cases.

For low $\left(s \ll s_{0}\right)$ and high $\left(s \gg s_{0}\right)$ values of $s$, where $s_{0}=0.282$ is the solution to the transcendental equation $0.869=$ $s_{0}^{1 / 3} \mathrm{e}^{s_{0}}$, one has

$$
\begin{aligned}
I_{L}\left(f_{L}\left(1+x_{L}^{2}\right)\right. & \ll 0.282) \\
& \simeq 1.151 H_{0} s_{L}^{1 / 3}=1.151 H_{0} f_{L}^{1 / 3}\left(1+x_{L}\right)^{2 / 3},
\end{aligned}
$$

and

$$
\begin{aligned}
I_{L}\left(f_{L}\left(1+x_{L}^{2}\right)\right. & \gg 0.282) \\
& \simeq H_{0} \exp \left(-s_{L}\right)=H_{0} \exp \left(-f_{L}\left(1+x_{L}\right)^{2}\right)
\end{aligned}
$$

in the linear cooling case, while

$$
\begin{aligned}
I_{N L}\left(f_{N L}\left(1+x_{N L}\right)^{5 / 4}\right. & \ll 0.282) \\
& \simeq 1.151 J_{0} f_{N L}^{1 / 3}\left(1+x_{N L}\right)^{1 / 6},
\end{aligned}
$$

and

$$
\begin{aligned}
I_{N L}\left(f_{N L}\left(1+x_{N L}\right)^{5 / 4}\right. & \gg 0.282) \\
& \simeq \frac{J_{0}}{\left(1+x_{N L}\right)^{1 / 4}} \exp \left(-f_{N L}\left(1+x_{N L}\right)^{5 / 4}\right)
\end{aligned}
$$

in the nonlinear cooling case, respectively.

In Figs. 1 and 2, we present the synchrotron spectra at different times $x=0,1,5,10$ for the linear and nonlinear cases, respectively. In the linear case, the maximum value of the intensity occurs at the frequency $f_{L, \max }=0.282 /\left(1+x_{L}\right)^{2}$, which 


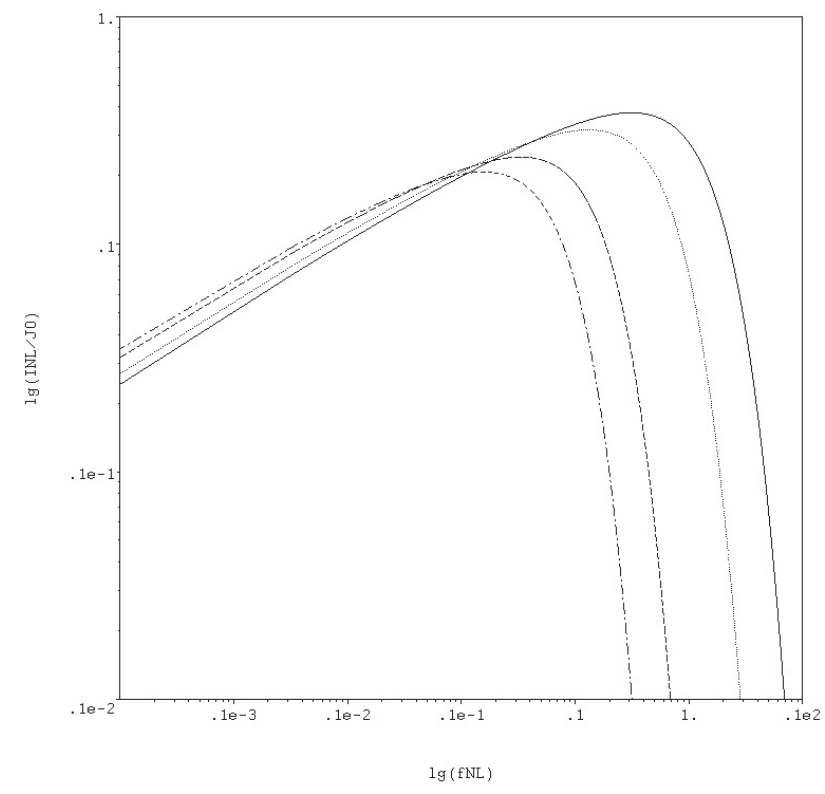

Fig. 2. Synchrotron intensity $I_{N L} / J_{0}$ as a function of the normalised frequency $f_{N L}$ in the nonlinear cooling case at four different times $x_{N L}=0$ (full curve), $x_{N L}=1$ (dotted curve), $x_{N L}=5$ (dashed curve), and $x_{N L}=10$ (dotted-dashed curve). The maximum intensity occurs at the frequency $f_{N L \text {, max }}=0.282 /\left(1+x_{N L}\right)^{5 / 4}$, which decreases $\propto 0.282 x_{N L}^{-5 / 4}$ at large times. Because of the decreasing magnetic field strength in equipartition conditions, the maximum intensity itself decreases as $\propto x_{N L}^{-1 / 4}$ with increasing time.

decreases $\propto 0.282 x_{L}^{-2}$ for large times $x_{L} \gg 1$. However, the maximum intensity is independent of time. The nonlinear case exhibits a different behaviour. Here the maximum intensity occurs at the frequency $f_{N L, \max }=0.282 /\left(1+x_{N L}\right)^{5 / 4}$, which decreases $\propto 0.282 x_{N L}^{-5 / 4}$ at large times. Also the maximum intensity itself decreases as $\propto x_{N L}^{-1 / 4}$ with increasing time.

In Figs. 3 and 4, we show the synchrotron light curves at different frequencies for the two cases. In the linear case at frequencies below $f_{L} \ll 0.282$, one has a transition from a power-law increase to a Gaussian decay at $x_{L}=0.53 / \sqrt{f_{L}}$ :

$$
\begin{aligned}
& \frac{I_{L}\left(x_{L}\right)}{I_{L}\left(x_{L}=0\right)} \simeq \\
& \begin{cases}\left(1+x_{L}\right)^{2 / 3} & \text { for } x_{L} \ll 0.53 / f_{L}^{1 / 2} \\
\exp \left[-f_{L}\left(x_{L}^{2}+2 x_{L}\right)\right] & \text { for } x_{L} \gg 0.53 / f_{L}^{1 / 2}\end{cases}
\end{aligned}
$$

The width of the Gaussian increases $\propto f_{L}^{-1 / 2}$ with decreasing frequency (see Fig. 3).

In the nonlinear case at frequencies below $f_{N L} \ll 0.282$, there is a transition from a weaker power-law increase to a nonGaussian decay at $x_{N L}=\left(0.282 / f_{L}\right)^{4 / 5}$

$$
\begin{aligned}
& \frac{I_{N L}\left(x_{N L}\right)}{I_{N L}\left(x_{N L}=0\right)} \simeq \\
& \begin{cases}\left(1+x_{N L}\right)^{1 / 6} & \text { for } x_{N L} \ll 0.363 f_{N L}^{-0.8} \\
x_{N L}^{-1 / 4} \exp \left[-f_{N L} x_{N L}^{5 / 4}\right] & \text { for } x_{N L} \gg 0.363 f_{N L}^{-0.8} .\end{cases}
\end{aligned}
$$

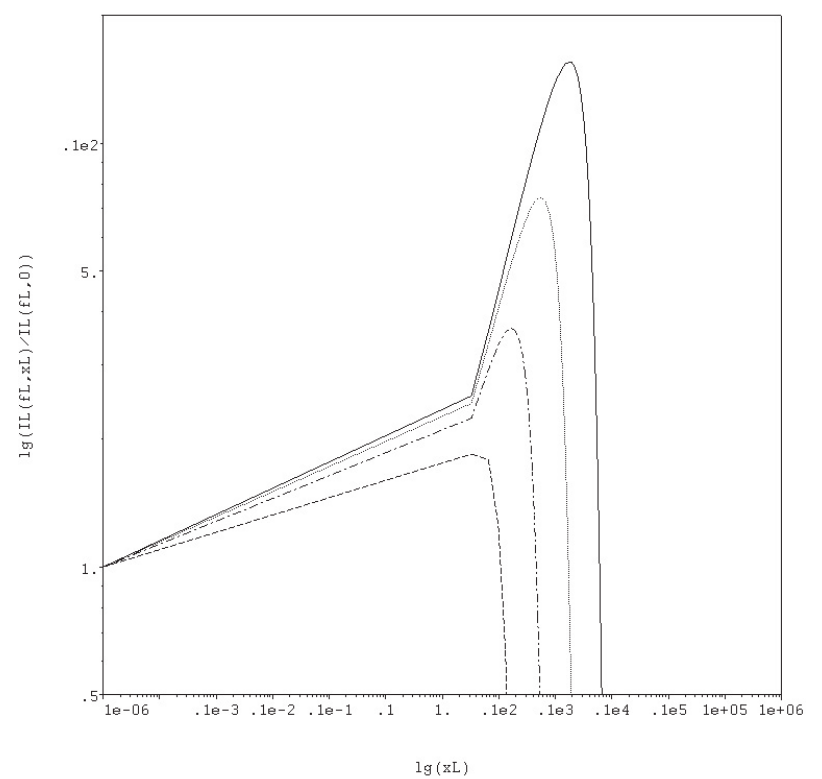

Fig. 3. Normalised synchrotron light curves $I_{L}\left(x_{L}\right) / I_{L}(x=0)$ as a function of the normalised time $x_{L}$ in the linear cooling case at four different normalised frequencies $f_{L}=10^{-5}$ (full curve), $f_{L}=10^{-4}$ (dotted curve), $f_{L}=10^{-3}$ (dotted-dashed curve), and $f_{L}=10^{-2}$ (dashed curve). The light curve exhibits a transition from a power-law increase to a Gaussian decay at $x_{L}=0.53 / \sqrt{f_{L}}$.

\subsection{Optically thin synchrotron fluences}

Finally we calculate the time-integrated synchrotron fluences

$F(f)=\int_{0}^{\infty} \mathrm{d} t I(f, t)$

as a function of the normalized frequencies in the linear and nonlinear cases. The intensities (21) and (22) immediately yield, after obvious substitutions,

$$
\begin{aligned}
F_{L}\left(f_{L}\right) & =H_{0} t_{1 / 2}^{L} \int_{0}^{\infty} \mathrm{d} x_{L} s_{L} C S\left(s_{L}\right) \\
& =\frac{H_{0} t_{1 / 2}^{L}}{2 f_{L}^{1 / 2}} \int_{f_{L}}^{\infty} \mathrm{d} s s^{1 / 2} C S(s)
\end{aligned}
$$

and

$$
\begin{aligned}
F_{N L}\left(f_{N L}\right) & =\frac{J_{0} t_{1 / 2}^{N L}}{3} \int_{0}^{\infty} \mathrm{d} x_{N L}\left(1+x_{N L}\right)^{-1 / 4} s_{N L} C S\left(s_{N L}\right) \\
& =\frac{4 J_{0} t_{1 / 2}^{N L}}{15 f_{N L}^{3 / 5}} \int_{f_{N L}}^{\infty} \mathrm{d} s s^{3 / 5} C S(s),
\end{aligned}
$$

respectively.

We use the asymptotic expansions (Crusius \& Schlickeiser 1988)

$C S(s) \simeq \begin{cases}a_{0} s^{-2 / 3} & \text { for } s \ll 1 \\ s^{-1} \mathrm{e}^{-s} & \text { for } s \gg 1,\end{cases}$

where $a_{0}=1.151275$, to derive the asymptotic behaviour of the two integrals for low and high normalised frequencies. For $f_{L} \ll$ 1 we obtain

$$
\begin{aligned}
\int_{f_{L}}^{\infty} \mathrm{d} s s^{1 / 2} C S(s) & =\int_{0}^{\infty} \mathrm{d} s s^{1 / 2} C S(s)-\int_{0}^{f_{L}} \mathrm{~d} s s^{1 / 2} C S(s) \\
& \simeq c_{0}-a_{0} \int_{0}^{f_{L}} \mathrm{~d} s s^{-1 / 6}=c_{0}-\frac{6 a_{0}}{5} f_{L}^{5 / 6}
\end{aligned}
$$




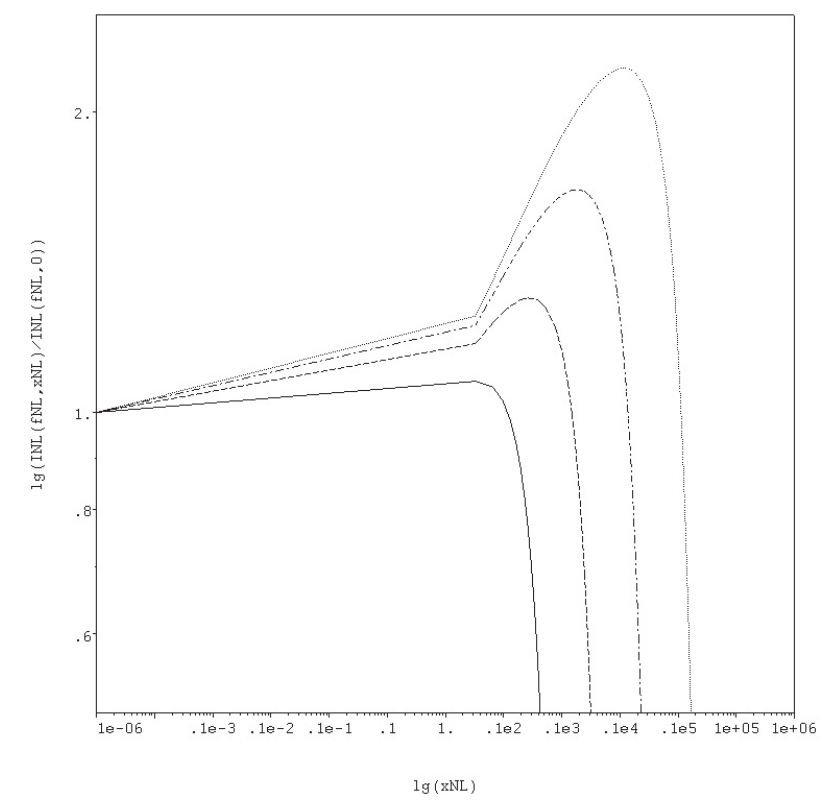

Fig. 4. Normalised synchrotron light curves $I_{N L}(x) / I_{L}(x=0)$ as a function of the normalised time $x_{N L}$ in the nonlinear cooling case at four different normalised frequencies $f_{N L}=10^{-5}$ (dotted curve), $f_{N L}=10^{-4}$ (dotted-dashed curve), $f_{N L}=10^{-3}$ (dashed curve), and $f_{N L}=10^{-2}$ (full curve). The light curve exhibits a transition from a weaker power-law increase to a non-Gaussian decay at $x_{N L}=\left(0.282 / f_{L}\right)^{4 / 5}$.

with the constant

$$
\begin{aligned}
c_{0} & =\int_{0}^{\infty} \mathrm{d} s s^{1 / 2} C S(s) \\
& =\frac{\Gamma\left(\frac{25}{12}\right) \Gamma\left(\frac{5}{12}\right)}{10 \pi^{3 / 2}}\left[40 \Gamma^{2}\left(\frac{3}{4}\right)-\frac{3 \pi}{\Gamma^{2}\left(\frac{5}{4}\right)}\right] .
\end{aligned}
$$

Likewise for $f_{N L} \ll 1$, we find

$\int_{f_{N L}}^{\infty} \mathrm{d} s s^{3 / 5} C S(s) \simeq c_{1}-\frac{15 a_{0}}{14} f_{N L}^{14 / 15}$

with the constant

$$
\begin{aligned}
c_{1} & =\int_{0}^{\infty} \mathrm{d} s s^{3 / 5} C S(s) \\
& =\frac{\Gamma\left(\frac{32}{15}\right) \Gamma\left(\frac{7}{15}\right)}{\pi \Gamma\left(\frac{8}{5}\right)}\left[2^{6 / 5} \Gamma^{2}\left(\frac{4}{5}\right)-\frac{8}{13} \frac{\Gamma^{2}\left(\frac{8}{5}\right)}{\Gamma^{2}\left(\frac{13}{10}\right)}\right] .
\end{aligned}
$$

For high frequencies $f_{L} \gg 1$ and $f_{N L} \gg 1$,

$\int_{f_{L}}^{\infty} \mathrm{d} s s^{1 / 2} C S(s) \simeq \int_{f_{L}}^{\infty} \mathrm{d} s s^{-1 / 2} \mathrm{e}^{-s} \simeq f_{L}^{-1 / 2} \mathrm{e}^{-f_{L}}$

and

$\int_{f_{N L}}^{\infty} \mathrm{d} s s^{3 / 5} C S(s) \simeq \int_{f_{N L}}^{\infty} \mathrm{d} s s^{-2 / 5} \mathrm{e}^{-s} \simeq f_{N L}^{-2 / 5} \mathrm{e}^{-f_{L}}$.

To leading orders, the fluences (31) and (32) vary with frequency as

$F_{L}\left(f_{L}\right)=\frac{H_{0} t_{1 / 2}^{L}}{2} \begin{cases}c_{0} f_{L}^{-1 / 2} & \text { for } f_{L} \ll 1 \\ f_{L}^{-1} \mathrm{e}^{-f_{L}} & \text { for } f_{L} \gg 1\end{cases}$ and

$$
F_{N L}\left(f_{N L}\right)=\frac{4 J_{0} t_{1 / 2}^{N L}}{15} \begin{cases}c_{1} f_{N L}^{-3 / 5} & \text { for } f_{N L} \ll 1 \\ f_{N L}^{-1} \mathrm{e}^{-f_{N L}} & \text { for } f_{N L} \gg 1\end{cases}
$$

At high normalised frequencies both fluences exhibit the same exponential cut-off, whereas at low normalised frequencies, the nonlinear fluence shows a steeper (by $\delta \alpha=0.1$ ) power-law behaviour $\left(\propto f_{N l}^{-0.6}\right)$ than the linear fluence $\left(\propto f_{N l}^{-0.5}\right)$. Note that the normalisations are different in both cases (see Eqs. (18) and (19)).

The predictions of the optically thin fluence behaviour with frequency, and the spectral intensity behaviour with both time and frequency provide conclusive tests for the presence of linear or nonlinear cooling in flaring nonthermal sources.

\section{Summary and conclusions}

In powerful cosmic nonthermal radiation sources with dominant magnetic-field self generation, the generation of magnetic fields at almost equipartition strength by relativistic plasma instabilities operates as fast as or faster than the acceleration or injection of ultra-high energy radiating particles in these sources. Consequently, the magnetic field strength becomes time-dependent and adjusts itself to the actual kinetic energy density of the radiating particles in these sources. We demonstrate that this coupling of the magnetic field and the magneticfield energy density to the kinetic energy of the particles changes both the synchrotron emissivity and the intrinsic temporal evolution of the relativistic electron energy spectrum after injection. In blazar and GRB sources, the nonlinear synchrotron cooling of each particle under equipartition conditions then is orders of magnitude quicker if more electrons are injected in the source. Such collective behaviour is completely different from the cooling behaviour in constant magnetic field strength sources. This dramatic reduction of the intrinsic radiation loss time may be essential to meet the observed rapid time variation (days) in the case of the non-blazar radio galaxy M 87. It also accords with the strong constraints on the required time dilatation from Doppler boosting for flaring blazar jets such as PKS 2155-304.

For the illustrative case of instantaneous injection of monoenergetic particles, we solved the nonlinear kinetic equation for the intrinsic temporal evolution of the relativistic particles. Unlike the linear case, the case of instantaneous injection of monoenergetic particles does not serve as a Green's function for the nonlinear kinetic equation due to the nonlinear dependence of the synchrotron loss term on an energy integral of the particle number density. In the case of relativistic electrons from the nonlinear electron density, we calculated also the corresponding optically thin synchrotron radiation intensity taking into account the time-dependence of the magnetic field strength under equipartition conditions. The comparison with the synchrotron intensity from the standard solution of the linear kinetic equation shows significant differences both in the spectral distributions at different times and the synchrotron light curves at different frequencies. Spectral differences also occur in the frequency distribution of the linear and nonlinear synchrotron fluences. These predictions of spectral behaviour with time and frequency provide conclusive tests for the presence or absence of linear or nonlinear cooling in flaring nonthermal sources.

Acknowledgements. We thank Dipl.-Phys. C. Röken for help with the graphical illustrations. We also thank the referee for useful comments that helped to improve the manuscript. This work was partially supported 
by the German Ministry for Education and Research (BMBF) through Verbundforschung Astroteilchenphysik grant 05 CH5PC1/6 and the Deutsche Forschungsgemeinschaft through grant Schl 201/16-2.

\section{Appendix A: Solution of the electron kinetic equation for linear cooling}

Inserting Eq. (4) tp the kinetic Eq. (6) yields

$\frac{\partial n(\gamma, t)}{\partial t}-D_{0} \frac{\partial}{\partial \gamma}\left[\gamma^{2} n(\gamma, t)\right]=q_{0} \delta\left(\gamma-\gamma_{0}\right) \delta(t)$

with the constant rate (5). Substituting $\tau=D_{0} t$ and setting $\gamma^{2} n=$ $R$ readily yields

$\frac{\partial R}{\partial \tau}-\gamma^{2} \frac{\partial R}{\partial \gamma}=q_{0} \gamma^{2} \delta\left(\gamma-\gamma_{0}\right) \delta(\tau)$.

With the new variable

$x=1 / \gamma$

Eq. (43) reads $\left(x_{0}=1 / \gamma_{0}\right)$

$\frac{\partial R}{\partial \tau}+\frac{\partial R}{\partial x}=q_{0} \delta\left(x-x_{0}\right) \delta(\tau)$

which is immediately solved by a Laplace transformation with respect to $\tau$ and the boundary condition requirement $R(\gamma=$ $\infty, t)=R(x=0, t)=0$. We find

$R(x, \tau)=q_{0} H\left[x-x_{0}\right] \delta\left(\tau+x_{0}-x\right)$,

where $H[x]$ denotes Heaviside's step function, yielding the solution (7).

\section{Appendix B: Solution of electron kinetic equation for nonlinear cooling}

Under equipartition conditions, the kinetic equation reads

$\frac{\partial n}{\partial t}-A_{0}\left[\int_{0}^{\infty} \mathrm{d} \gamma \gamma n\right] \frac{\partial}{\partial \gamma}\left(\gamma^{2} n\right)=q_{0} \delta\left(\gamma-\gamma_{0}\right) \delta(t)$

with the abbreviation constant $A_{0}$. We substitute $y=A_{0} t$ and set $S=\gamma^{2} n$ to obtain

$\frac{\partial S}{\partial y}-\left[\int_{0}^{\infty} \mathrm{d} \gamma S / \gamma\right] \gamma^{2} \frac{\partial S}{\partial \gamma}=q_{0} \gamma^{2} \delta\left(\gamma-\gamma_{0}\right) \delta(y)$.

Again we use $x=1 / \gamma$ as an independent variable, so that with $x_{0}=1 / \gamma_{0}$

$\frac{\partial S}{\partial y}+\frac{\partial S}{\partial x}\left[\int_{0}^{\infty} \mathrm{d} x x^{-1} S(x, y)\right]=q_{0} \delta\left(x-x_{0}\right) \delta(y)$.

Now we define $T$ through

$\frac{\mathrm{d} T}{\mathrm{~d} y}=U(y)=\int_{0}^{\infty} \mathrm{d} x x^{-1} S(x, y)$

so that

$T(y)=\int_{0}^{y} \mathrm{~d} b U(b)$.
Then Eq. (49) is just

$\frac{\partial S}{\partial T}+\frac{\partial S}{\partial x}=q_{0} \delta\left(x-x_{0}\right) \delta(T)$,

which is solved by the method of characteristics: set $x-T=\xi$ (i.e. $x=T+\xi$ ), implying

$\left.\frac{\partial}{\partial T}\right|_{x}=\left.\frac{\partial}{\partial T}\right|_{\xi}+\frac{\partial}{\partial \xi} \frac{\partial \xi}{\partial T}=\left.\frac{\partial}{\partial T}\right|_{\xi}-\frac{\partial}{\partial \xi} ;\left.\quad \frac{\partial}{\partial x}\right|_{T}=\frac{\partial}{\partial \xi}$,

so that Eq. (52) becomes

$\frac{\partial S}{\partial T}=q_{0} \delta\left(T+\xi-x_{0}\right) \delta(T)$

with the solution

$$
\begin{aligned}
S(x, T) & =S_{h}(\xi)+q_{0} \int^{T} \mathrm{~d} w \delta\left(w+\xi-x_{0}\right) \delta(w) \\
& =S_{h}(x-T)+q_{0} H[T] \delta\left(x-T-x_{0}\right),
\end{aligned}
$$

where $S_{h}(\xi)$ is any solution of $\xi$ determined by the boundary condition $S(x=0, T)=0$. We derive

$S_{h}(-T)=-q_{0} H[T] \delta\left(-T-x_{0}\right)$

or

$S_{h}(z)=-q_{0} H[-z] \delta\left(z-x_{0}\right)$.

The solution (54) thus becomes

$S(x, T)=q_{0} \delta\left(x-T-x_{0}\right)(H[T]-H[T-x])$.

The final step is then to explicitly calculate the time variable $T$ as a function of $y$. Use Eq. (56) in Eq. (50) to write

$$
\begin{aligned}
\frac{\mathrm{d} T}{\mathrm{~d} y} & =U=\int_{0}^{\infty} \mathrm{d} x x^{-1} S(x, y) \\
& =q_{0} \int_{0}^{\infty} \mathrm{d} x x^{-1} \delta\left(x-T-x_{0}\right)(H[T]-H[T-x]) \\
& =q_{0} H[T] H\left[x_{0}\right] \frac{1}{x_{0}+T} .
\end{aligned}
$$

With $T=0$ for $y=0$ Eq. (57) readily yields

$q_{0} y=\frac{T^{2}}{2}+x_{0} y$

or

$T(y)=\sqrt{2 q_{0} y+x_{0}^{2}}-x_{0}=\sqrt{2 q_{0} A_{0} t+x_{0}^{2}}-x_{0}$.

In terms of $y$, the solution (56) reads

$$
\begin{aligned}
S(x, y)= & q_{0} \delta\left(x-\sqrt{2 q_{0} y+x_{0}^{2}}\right)\left(H\left[\sqrt{2 q_{0} y+x_{0}^{2}}-x_{0}\right]\right. \\
& \left.-H\left(\sqrt{2 q_{0} y+x_{0}^{2}}-x_{0}-x\right]\right) \\
= & q_{0} \delta\left(x-\sqrt{2 q_{0} y+x_{0}^{2}}\right)\left(H\left[x-x_{0}\right]-H\left[-x_{0}\right]\right) .
\end{aligned}
$$


With $x_{0}>0$ we have

$S\left(x, x_{0}, t\right)=q_{0} H\left[x-x_{0}\right] \delta\left(x-\sqrt{2 q_{0} A_{0} t+x_{0}^{2}}\right)$,

giving for the nonlinear solution

$$
\begin{aligned}
n_{N L}\left(\gamma, \gamma_{0}, t\right) & =\frac{q_{0}}{\gamma^{2}} H\left[\gamma_{0}-\gamma\right] \delta\left(\gamma^{-1}-\sqrt{2 A_{0} q_{0} t+\gamma_{0}^{-2}}\right) \\
& =q_{0} H\left[\gamma_{0}-\gamma\right] \delta\left(\gamma-\gamma_{N L}(t)\right)
\end{aligned}
$$

in agreement with Eq. (12).

\section{Appendix C: Optically thin synchrotron radiation}

In both cases of linear and nonlinear cooling, the electron spectrum is represented as $n(\gamma, t)=q_{0} H\left[\gamma_{0}-\gamma\right] \delta\left(\gamma-\gamma_{1}(t)\right)$ with $\gamma_{1}(t)$ given by Eqs. (5) and (14), so that the synchrotron intensity (17) can be written as

$I(v, t)=\frac{L P_{0} q_{0}}{4 \pi} \frac{v}{\gamma_{1}^{2}(t)} C S\left[\frac{2 v}{3 v_{0} \gamma_{1}^{2}(t)}\right]$.

In the linear cooling case, we obtain

$$
\begin{aligned}
I_{L}(v, t) & =\frac{3 L q_{0} P_{0} v_{0}}{8 \pi} \frac{v}{v_{L}(t)} C S\left[\frac{v}{v_{L}(t)}\right] \\
& \simeq \frac{3 L q_{0} P_{0} v_{0}}{8 \pi}\left(0.869\left(\frac{v_{L}(t)}{v}\right)^{1 / 3}+\exp \left[\frac{v}{v_{L}(t)}\right]\right)^{-1}
\end{aligned}
$$

with the characteristic frequency

$$
\begin{aligned}
v_{L}(t) & =\frac{3}{2} v_{0}\left(\gamma_{L}\right)^{2}=\frac{3}{2} \frac{v_{0} \gamma_{0}^{2}}{\left(1+D_{0} \gamma_{0} t\right)^{2}} \\
& =\frac{3}{2} \frac{v_{0} \gamma_{0}^{2}}{\left(1+\left(t / t_{1 / 2}^{L}\right)\right)^{2}} .
\end{aligned}
$$

In the nonlinear cooling case an additional time dependence results from the time-varying magnetic field (2) so that the electron gyrofrequency is

$$
\begin{aligned}
v_{0}(t) & =\frac{e B(t)}{2 \pi m_{\mathrm{e}} c}=c \sqrt{2 \pi^{-1} e_{B} r_{0}}\left[\int_{0}^{\infty} \gamma n_{N L}(\gamma, t)\right]^{1 / 2} \\
& =c \sqrt{2 e_{B} r_{0} q_{0} \gamma_{N L}(t) / \pi},
\end{aligned}
$$

where $r_{0}=2.82 \times 10^{-13} \mathrm{~cm}$ is the classical electron radius and where we have inserted the nonlinear solution (11). We find for the nonlinear optical synchrotron intensity

$$
\begin{aligned}
I_{N L}(v, t)= & \frac{3 L P_{0} q_{0} c}{8 \pi} \sqrt{2 e_{B} q_{0} r_{0} / \pi} \gamma_{N L}^{1 / 2}(t) \frac{v}{v_{N L}(t)} C S\left[\frac{v}{v_{N L}(t)}\right] \\
\simeq & \frac{3 L P_{0} q_{0} c}{8 \pi} \sqrt{2 e_{B} q_{0} r_{0} / \pi} \gamma_{N L}^{1 / 2}(t) \\
& \times\left(0.869\left(\frac{v_{N L}(t)}{v}\right)^{1 / 3}+\exp \left[\frac{v}{v_{N L}(t)}\right]\right)^{-1}
\end{aligned}
$$

with the nonlinear characteristic frequency

$$
\begin{aligned}
v_{N L}(t) & =\frac{3}{2} c \sqrt{2 e_{B} q_{0} r_{0} / \pi} \gamma_{N L}^{5 / 2}(t) \\
& =c \sqrt{\frac{3}{2}} c \sqrt{2 e_{B} q_{0} r_{0} / \pi} \frac{\gamma_{0}^{5 / 2}}{\left[1+2 A_{0} q_{0} \gamma_{0}^{2} t\right]^{5 / 4}} \\
& =\frac{3}{2} c \sqrt{2 e_{B} q_{0} r_{0} / \pi} \frac{\gamma_{0}^{5 / 2}}{\left[1+\left(3 t / t_{1 / 2}^{N L}\right)\right]^{5 / 4}} .
\end{aligned}
$$

\section{References}

Aharonian, F. A., Akhperjanian, A. G., Bazer-Bachi, A. R., et al. (H.E.S.S. Collaboration) 2006, Science, 314, 1424

Aharonian, F. A., Akhperjanian, A. G., Bazer-Bachi, A. R., et al. (H.E.S.S. Collaboration) 2007, ApJ, 664, L71

Beck, R., \& Krause, M. 2005, AN, 326, 414

Böttcher, M. 2007, Astroph. \& Space Sci., 309, 95

Böttcher, M., \& Chiang, J. 2002, ApJ, 581, 127

Böttcher, M., \& Reimer, A. 2004, ApJ, 609, 576

Crusius, A., \& Schlickeiser, R. 1986, A\&A, 164, L16

Crusius, A., \& Schlickeiser, R. 1988, A\&A, 196, 327

Dermer, C. D., \& Schlickeiser, R. 1992, Science, 257, 1642

Dermer, C. D., \& Schlickeiser, R. 2002, ApJ, 575, 667

Frail, D. A., Waxman, E., \& Kulkarni, S. R. 2000, ApJ, 537, 191

Foschini, L., Ghisellini, G., Tavecchio, F., et al. 2007, ApJ, 657, L81

Frederiksen, J. T., Hededal, C. B., Haugbolle, T., \& Nordlund, A. 2004, ApJ, 608, L13

Jaroschek, C., Lesch, H., \& Treumann, R. 2005, ApJ, 618, 822

Kapetanakos, C. A. 1974, Appl. Phys. Lett., 25, 484

Kardashev, N. S. 1962, Sov. Astron., 6, 317

Kino, M., Takahara, F., \& Kusunose, M. 2002, ApJ, 564, 97

Lee, R., \& Lampe, M. 1973, Phys. Rev. Lett., 31, 1390

Medvedev, M. V., \& Loeb, A. 1999, ApJ, 526, 697

Medvedev, M. V., Lazzati, D., Morsony, B. C., \& Workman, J. C. 2007, ApJ, 666,339

Meszaros, P., \& Rees, M. J. 1993, ApJ, 405, 278

Meszaros, P., \& Rees, M. J. 1997, ApJ, 476, 232

Moiseev, S. S., \& Sagdeev, R. Z. 1963, J. Nucl. Energy, 5, 63

Montgomery, D. C., \& Joyce, G. 1969, J. Plasma Phys., 4, 1

Ng, J. S. T., \& Noble, R. J. 2006, Phys. Rev. Lett., 96, 115006

Nishikawa, K. I., Hardee, P., Richarson, G., et al. 2003, ApJ, 595, 555

Paczynski, B., \& Rhoads, J. E. 1993, ApJ, 418, L5

Panaitescu, A., \& Kumar, P. 2000, ApJ, 543, 66

Piran, T. 1999, Phys. Rep., 314, 575

Piran, T. 2005, Rev. Mod. Phys., 76, 143

Reimer, A., Protheroe, R. J., \& Donea, A. 2004, New Astr. Rev., 48, 411

Sari, R., Piran, T., \& Narayan, R. 1998, ApJ, 497, L17

Sakai, J.-I., Schlickeiser, R., \& Sukla, P. K. 2004, Phys. Lett. A, 330, 384

Schamel, H. 1972, Plasma Phys., 14, 905

Schlickeiser, R. 2003, A\&A, 420, 397

Schlickeiser, R., Sievers, A., \& Thiemann, H. 1988, A\&A, 182, 21

Schlickeiser, R., Vainio, R., Böttcher, M., et al. 2002, A\&A, 393, 69

Silva, L. O., Fonseca, R. A., Tonge, J. W., et al. 2003, ApJ, 596, L121

Stockem, A., Lerche, I., \& Schlickeiser, R. 2007, ApJ, 659, 419

Tatarakis, M., et al. 2003, Phys. Rev. Lett., 90, 175001

Van der Horst, A. J., Kamble, A., Resmi, L., et al. 2007, A\&A, submitted, [astro-ph 0706.1321]

Van Paradijs, J., Kouveliotou, C., \& Wijers, R. 2000, ARA\&A, 38, 379

Waxman, E. 2006, Plasma Phys. Controll. Fusion, 48, B137

Workman, J. C., Morsony, B. C., Lazzati, D., \& Medvedev, M. V. 2007, MNRAS, in press, [astro-ph/0703420]

Zhang, Y. H., Treves, A., Celotti, A., et al. 2002, ApJ, 572, 762 